191 ISOKINETIC DEFICITS AT 6 MONTHS AFTER ACL RECONSTRUCTION INFLUENCE THE RATE OF REINJURIES AND ACTIVITY LEVEL

1,2,3Renato Andrade, ${ }^{1}$ José Dias, ${ }^{1}$ Cátia Cardoso, ${ }^{2}$ Cristina Valente, $1,2,3,4$ Rogério Pereira, 1,2,5,6,7 Alexandre Rebelo-Marques, ${ }^{8}$ Tiago Proença, ${ }^{8}$ Pedro Lamas, ${ }^{12,13}$ Nuno Cordeiro, ${ }^{9}$ Alcindo Silva, $1,2,10,11 \mathrm{~J}$ oão Espregueira-Mendes. ${ }^{1}$ Clínica do Dragão, Espregueira-Mendes Sports Centre - FIFA Medical Centre of Excellence, Porto, Portugal; ${ }^{2}$ Dom Henrique Research Centre, Porto, Portugal; ${ }^{3}$ Faculty of Sports, University of Porto, Porto, Portugal; ${ }^{4}$ Escola Superior de Saúde, Universidade Fernando Pessoa, Porto, Portugal; ${ }^{5}$ Faculty of Medicine, University of Coimbra, Coimbra, Portugal; ${ }^{6}$ Clinical Academic Center of Coimbra, Coimbra, Portugal; ${ }^{7}$ Coimbra Institute for Clinical and Biomedical Research (iCBR), Faculty of Medicine, University of Coimbra, Coimbra, Portugal; ${ }^{8}$ Fisiocinética - Centro Isocinético, Porto, Portugal; ${ }^{9}$ Hospital da Luz - Arrábida, Vila Nova de Gaia, Portugal; ${ }^{10} /$ CVS/3B's-PT Government Associate Laboratory, Braga/Guimarães, Portugal; " ${ }^{11}$ Escola de Medicina, Universidade do Minho, Braga, Portugal; ${ }^{12}$ Instituto Politécnico de Castelo Branco, Castelo Branco, Portugali, ${ }^{13}$ AGE.COMM, Castelo Branco, Portugal

\subsection{6/bjsports-2021-IOC.176}

Background Recent evidence suggests that athletes may not be prepared to return to sport at 6 months following an anterior cruciate ligament (ACL) reconstruction.

Objective Identify knee isokinetic neuromuscular deficits at 6 months after ACL reconstruction and assess if deficits impact on the rate of new knee injuries and level of activity.

Design Retrospective study.

Setting Recreational and competitive athletes.

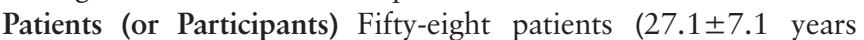
old; 79\% male) that underwent ACL reconstruction (62\% hamstrings and 38\% bone-patellar-tendon-bone grafts) and that performed the knee isokinetic testing at 6 months.

Interventions (or Assessment of Risk Factors) Bilateral knee isokinetic assessment at 6 months, 6 and 8 Con/Con repetitions, at $60 \%$ and $180 \%$, respectively.

Main Outcome Measurements Peak torque of knee extensors and flexors at $60 \%$ and $180 \%$ s and Con/Con unilateral ratio. Prevalence of bilateral $(>10 \%)$ and unilateral ratio $(<0.47$ and $>0.80$ ) abnormalities. The level of activity (Tegner) and number of new knee injuries (ACL or other knee injury).

Results Fifty-two participants (90\%) had bilateral deficits at $60 \%, 74 \%$ and $59 \%$ for knee extensors and flexors, respectively. Unilateral abnormalities were present on $16 \%$ of participants at $60 \%$ and $180 \%$ s. Thirty-seven participants had 2 or more years follow-up (28 hamstrings and 9 bone-patellar-tendon-bone graft). From these, there were 4 new ACL injuries (75\% contralateral) and 8 other knee injuries $(50 \%$ contralateral). Rate of new knee injuries was more frequent on hamstrings graft $(25 \%$ vs $10 \%)$. Those with bilateral deficits at $60 \%(\mathrm{~s}=31)$ had higher rate of new knee injuries $(23 \%$ vs $17 \%$ ) and higher rate of Tegner level decrease (45\% vs $17 \%)$. Conclusions We found an unacceptably high rate of participants (90\%) display knee isokinetic bilateral deficits at $60 \%$ at 6 months after ACL reconstruction. Bilateral deficits seem to influence the rate of new knee injuries and Tegner activity level.

\section{ASSESSMENTS FOR NEUROMUSCULAR CONTROL AFTER AN ANTERIOR CRUCIATE LIGAMENT INJURY TO DECIDE UPON RETURN TO SPORTS}

\footnotetext{
${ }^{1,2}$ Angela Blasimann, ${ }^{1}$ Irene Koenig, ${ }^{2}$ Isabel Baert, ${ }^{1}$ Heiner Baur, ${ }^{2}$ Dirk Vissers. ${ }^{1}$ Bern University of Applied Sciences, Department of Health Professions, Division of Physiotherapy, Bern, Switzerland; '2University of Antwerp, Faculty of Medicine and Health Sciences, Department of Rehabilitation Sciences and Physiotherapy, Antwerp, Belgium
}

Background Adequate neuromuscular control of the knee could be one element to prevent secondary injuries after an anterior cruciate ligament (ACL) injury. However, it is unclear which measurements should be used to assess neuromuscular control of the knee for a safe return to sports (RTS).

Objective To summarize assessments for neuromuscular control after an ACL injury to decide upon a safe RTS.

Design Systematic literature review, registered in PROSPERO (CRD42019122188).

Setting MEDLINE, EMBASE, CINAHL, Cochrane Library, SPORTDiscus, PEDro, and Web of Science were searched from inception to March 2019 and updated in November 2020. Risk of bias was assessed with a modified Downs \& Black checklist.

Patients (or Participants) Male or female adults after an ACL injury, either treated surgically or conservatively. Time from injury until the assessment should be at least six months and the participants should have medical clearance for RTS.

Interventions (or Assessment of Risk Factors) Assessments for neuromuscular control in ACL-injured adults compared to the contralateral limb or healthy controls during dynamic activities.

Main Outcome Measurements Outcome measures describing neuromuscular control of lower limb muscles in domains of time, amplitude or activity related to electromyography (EMG).

Results From initially 1388 hits, a total of 38 mainly crosssectional, case-controlled studies were included for qualitative analysis. Most studies provided surface EMG outcomes of thigh muscles during jumping, running or squatting. Outcomes measures described neuromuscular control of the knee in domains of time, amplitude or activity. Risk of bias was medium to high due to an unclear description of participants and prior interventions, confounding factors and incompletely reported results.

Conclusions Despite a wide range of EMG outcome measures for neuromuscular control, none was used to decide upon a safe RTS. Additional studies are needed to assess neuromuscular control in adult ACL patients to prevent secondary injuries.

\section{RISK FACTORS FOR CONTRA-LATERAL SECONDARY ANTERIOR CRUCIATE LIGAMENT INJURY: A SYSTEMATIC REVIEW WITH META-ANALYSIS}

${ }^{1,2}$ Anna Cronström, ${ }^{1}$ Tengman Eva, ${ }^{1} \mathrm{~K}$ Häger Charlotte. 'Department of Community Medicine and Rehabilitation, Umeå University, Umeå, Sweden; ${ }^{2}$ Department of Health Sciences, Lund University, Lund, Sweden

\subsection{6/bjsports-2021-IOC.178}

Background There is limited knowledge about which risk factors that contribute to the high numbers of contra-lateral anterior cruciate ligament (C-ACL) injury after primary ACL injury.

Objective To systematically review intrinsic risk factors for sustaining a C-ACL injury.

Design A systematic review with meta-analysis according to the PRISMA guidelines. Four databases (MEDLINE, CINAHL, EMBASE, Sport Discus) were searched from inception to January 2020. Meta-analyses were performed and expressed as odds ratios (OR). 